\title{
THE SPARC PROJECT: A HIGH BRIGHTNESS ELECTRON BEAM SOURCE AT LNF TO DRIVE A SASE-FEL EXPERIMENT
}

\author{
D.Alesini, S.Bertolucci, M.E.Biagini, C.Biscari, R.Boni, M.Boscolo, M.Castellano, A.Clozza, G.Di Pirro, A.Drago, \\ A.Esposito, M.Ferrario, V.Fusco, A.Gallo, A.Ghigo, S.Guiducci, M.Incurvati, C.Ligi, F.Marcellini, M.Migliorati, \\ C.Milardi, L.Palumbo, L.Pellegrino, M.Preger, P.Raimondi, R.Ricci, C.Sanelli, M.Serio, F.Sgamma, B.Spataro, \\ A.Stecchi, A.Stella, F.Tazzioli, C.Vaccarezza, M.Vescovi, C.Vicario, M.Zobov, INFN-Frascati
}

F.Alessandria, A.Bacci, I.Boscolo, F.Broggi, S.Cialdi, C.DeMartinis, D.Giove, C.Maroli, V.Petrillo, M.Romè, L.Serafini, INFN-Milano

D.Levi, M.Mattioli, G.Medici，INFN-Romal

L.Catani, E.Chiadroni, S.Tazzari, INFN-Roma2

\begin{abstract}
R.Bartolini, F.Ciocci, G.Dattoli, A.Doria, F.Flora, G.P.Gallerano, L.Giannessi, E.Giovenale, G.Messina, L.Mezi, P.L.Ottaviani, L.Picardi, M.Quattromini, A.Renieri, C.Ronsivalle, ENEA-Frascati
\end{abstract}

A.Cianchi, A.D’Angelo, R.Di Salvo, A.Fantini, D.Moricciani, C.Schaerf, Università Roma Tor Vergata

J.B. Rosenzweig, UCLA - Dept. of Physics and Astronomy

\begin{abstract}
The Project SPARC (Sorgente Pulsata e Amplificata di Radiazione Coerente), proposed by a collaboration among ENEA-INFN-CNR-Universita' di Roma Tor VergataINFM-ST, was recently funded by the Italian Government. The aim of the project is to promote an $\mathrm{R} \& \mathrm{D}$ activity oriented to the development of a coherent ultra-brilliant X-ray source in Italy (SPARX proposal [1]). The SPARC collaboration identified a program based on two main issues: the generation of ultra-high peak brightness electron beams and experimental study of SASE-FEL process with generation of resonant higher harmonics. The SPARC project is being designed in order to encompass the construction of an advanced photoinjector producing a $150-200 \mathrm{MeV}$ beam to drive a SASE-FEL in the optical range. The machine will be built at LNF, inside an underground bunker: it is comprised of an rf gun driven by a Ti:Sa laser, injecting into three SLAC accelerating sections. We foresee conducting investigations on the emittance correction[2] and on the rf compression techniques[3], which are expected to increase the peak current achievable at the injector exit up to $\mathrm{kA}$ level, with proper preservation of the transverse emittance. Although the system is expected to drive a FEL experiment, it can be used also to investigate beam physics issues like surface-roughness-induced wake fields, bunch-length measurements in the sub-ps range, emittance degradation in magnetic compressors due to CSR, and Compton backscattering production of sub-ps $\mathrm{X}$-ray pulses.
\end{abstract}

\section{PROJECT OVERVIEW}

The overall SPARC Project consists of 4 main lines of activity:
1) $150 \mathrm{MeV}$ Advanced Photo-Injector: the performances of X-ray SASE-FEL's are critically dependent on the peak brightness of the electron beam delivered at the undulator entrance. Two main issues to investigate: generation of the electron beam and compression via magnetic and/or velocity bunching.

2) SASE-FEL Visible-VUV Experiment: this is aimed to investigate the problems related to the beam matching into an undulator and the alignment with the radiation beam, as well as the generation of non-linear coherent higher harmonics. The SASE FEL experiment will be performed with the $150 \mathrm{MeV}$ beam, using a segmented undulator with additional strong focusing to observe FEL radiation at $500 \mathrm{~nm}$ and below.

3) X-ray Optics/Monochromators: the X-ray FEL radiation will provide unique radiation beams in terms of peak brightness and pulse time duration $(100 \mathrm{fs})$. This project will pursue a vigorous $R \& D$ activity on the analysis of radiation-matter interactions in the spectral range of SASE X-ray FEL's (from 0.1 to $10 \mathrm{~nm}$ ), and on the design of new optics and monochromators.

4) Soft X-ray table-top Source: to test these optics and start the R\&D on applications the project will undertake the upgrade of the presently operated table-top source of X-rays at INFM Politecnico Milano, delivering $10^{7}$ soft X-ray photons in 10-20 fs pulses by means of high harmonic generation in a gas. 


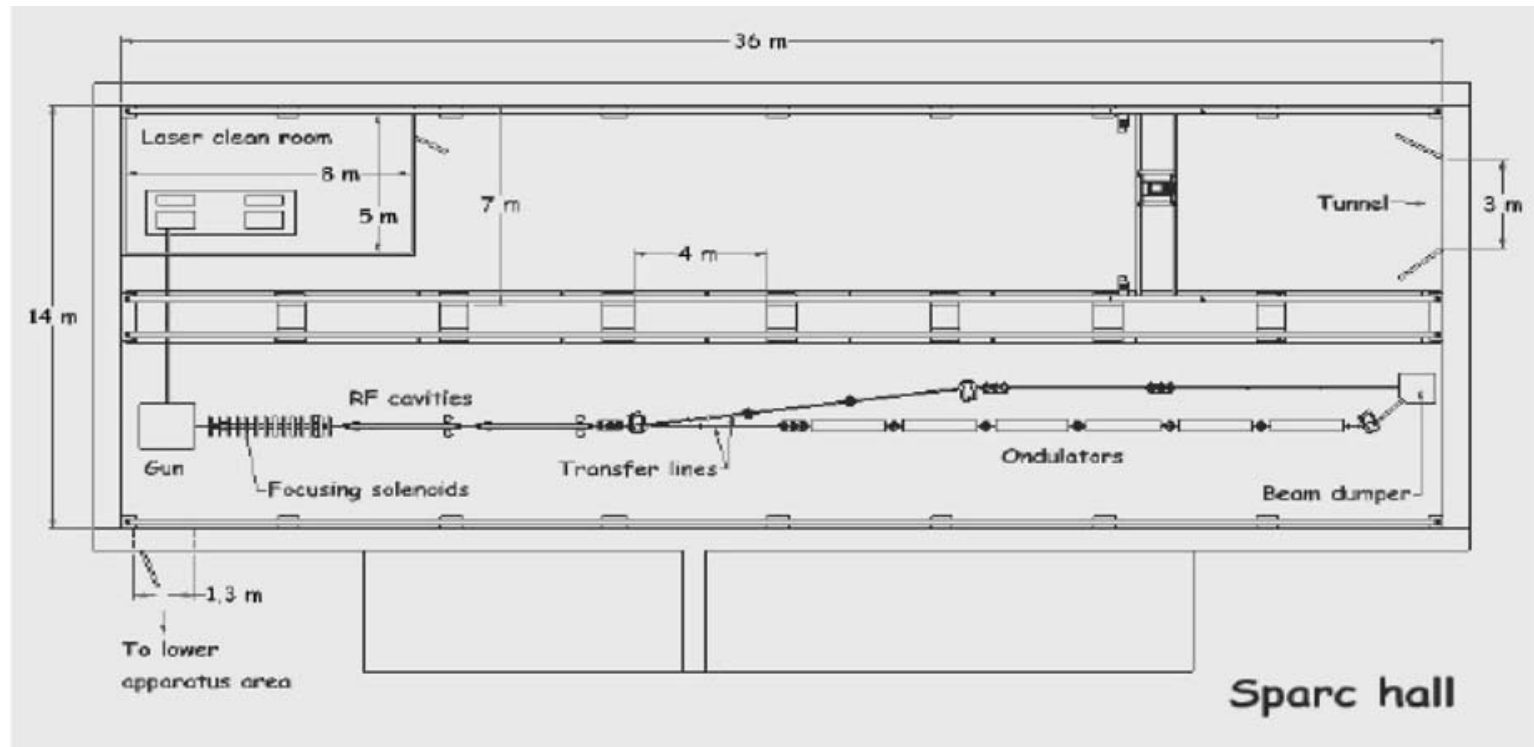

Figure 1: Lay-out of SPARC Advanced Photo-Injector and SASE-FEL Experiment

In the following we present an overview of the design for the system under construction at the Frascati National Laboratories of INFN, by a collaboration between two national research institutions, INFN and ENEA namely, aiming at reaching the scientific and technological goals indicated in the first two topics listed above, i.e. the production of a high brightness electron beam and the operation at saturation of a SASE-FEL experiment driven by such an electron beam.

\section{ADVANCED PHOTO-INJECTOR}

The main goals of this activity are the acquisition of expertise in the construction, commissioning and characterization of an advanced photo-injector system and the experimental investigation of two new ideas that have been recently conceived and presented by the study group: the optimum working point for high brightness RF photo-injectors and RF bunch compression technique.

The $150 \mathrm{MeV}$ injector will be built inside an available bunker of the Frascati INFN National Laboratories: the general layout of the system is shown in Fig. 1. The system consists of a 1.6 cell RF gun operated at S-band (2.856 GHz, of the BNL/UCLA/SLAC type) and high peak field on the cathode $(120 \mathrm{MeV} / \mathrm{m})$ with incorporated metallic photo-cathode $(\mathrm{Cu}$ or $\mathrm{Mg})$, generating a $6 \mathrm{MeV}$ beam[4]. The beam is then focused and matched into 3 accelerating sections of the SLAC type (S-band TW) which accelerates the bunch up to $150-200 \mathrm{MeV}$. For the Laser system it is planned to use the third harmonic of the radiation from a Ti:Sa laser with the oscillator pulse train locked to the RF . To obtain the time pulse shape we are going to test the manipulation of frequency lines in the large bandwidth of Ti:Sa, in order to produce the $10 \mathrm{ps}$ flat top shape. We can use a liquid crystal mask in the Fourier plane for nondispersive optic arrangement or a collinear acusto-optic modulator for line frequency manipulation. The goal of these tests is to obtain a pulse rise time shorter than 1 ps with intensity ripples along the 10 ps pulse smaller than $30 \%$ (peak to peak): under such a condition the beam emittance achievable at the end of the photo-injector is foreseen to be smaller than $1 \mu \mathrm{m}$.

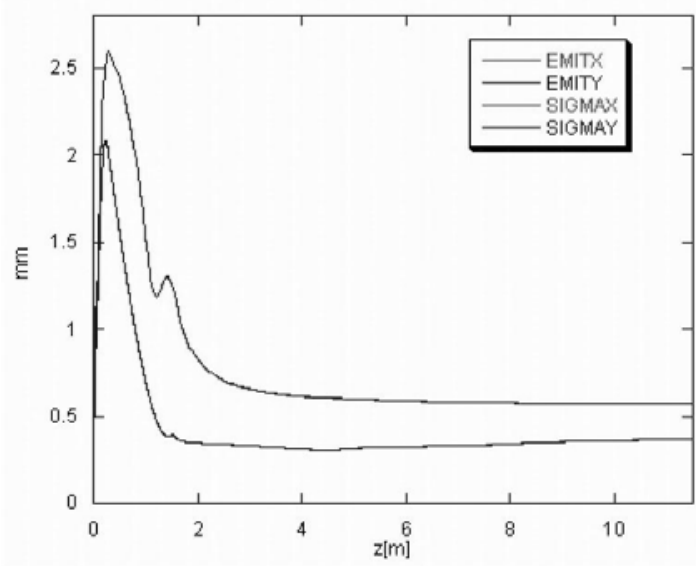

Figure 2: Parmela simulation of emittance and envelope evolution along the SPARC photo-injector (only two accelerating sections taken into account)

The first experiment is planned to verify the beam emittance compensation process. The key point is the measurement, at different bunch charges, of the emittance oscillation in the drift after the gun where a double minima behavior is expected. The optimum beam matching to the booster is predicted on the relative maximum, see Fig. 2. A dedicated movable emittance measurement station has been designed, as shown in Fig. 3. Our simulations using PARMELA indicate that we can generate in this way a beam as required by the FEL experiment at $150 \mathrm{MeV}$. The rms correlated energy 
spread is $0.2 \%$ with a rms norm. emittance lower than 2 $\mu \mathrm{m}$ (at $1 \mathrm{nC}$ bunch charge, 85 peak current). The slice energy spread and the slice norm. emittance, calculated over a $300 \mu \mathrm{m}$ slice length, are below $0.05 \%$ and $1 \mu \mathrm{m}$ respectively, all over the bunch. A complete investigation over the parameter range of the system is in progress, in particular tolerances and sensitivities are being considered in the beam dynamics simulations [5].

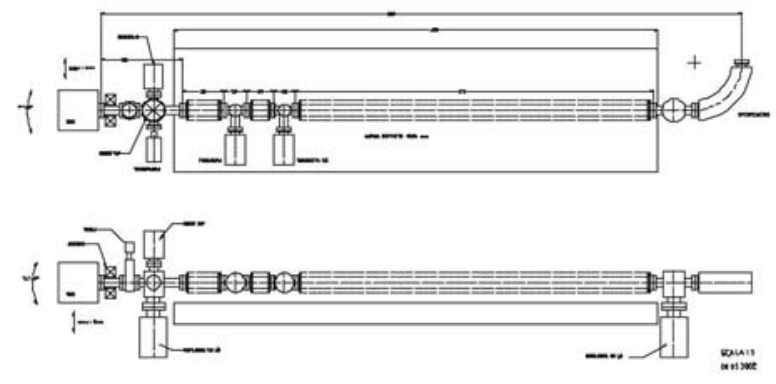

Figure 3: movable emittance measurement station. A pepperpot and a screen are connected with three bellows in order to scan the emittance along $1 \mathrm{~m}$ long drift

\section{SASE FEL EXPERIMENT}

The FEL SASE experiment will be conducted using a permanent magnet undulator made of 6 sections, each $2.13 \mathrm{~m}$ long, separated by $0.36 \mathrm{~m}$ gaps hosting single quadrupoles which focus in the horizontal plane. The undulator period is set at $3.0 \mathrm{~cm}$, with an undulator parameter $\mathrm{k}_{\mathrm{w}}=1.4$. A simulation performed with GENESIS is reported in Fig. 4, showing the exponential growth of the radiation power along the undulator. Almost 108 Watts can be reached after $14 \mathrm{~m}$ of total undulator length. Preliminary evaluations of the radiation power generated into the non-linear coherent odd higher harmonics show that $10^{7}$ and $7 \times 10^{5} \mathrm{~W}$ can be reached on the third and fifth harmonics, respectively.

\section{FURTHER EXPERIMENTS}

Two main upgrades will be implemented in a second phase of the project. A dedicated accelerating section will be inserted downstream the RF gun in order to exploit the full potentialities of the velocity bunching technique. Furthermore, in the parallel beam line a magnetic chicane will be installed to allow the experimental investigation of CSR induced effects on emittance degradation and surface roughness wake-field effects. Its design and construction will proceed in parallel to the commissioning of the SPARC injector system (RF gun +3 standard SLAC-type $3 \mathrm{~m}$ sections). These tests are of great relevance in our $R \& D$ program in view of the development of a coherent X-ray source according to the
SPARX proposal[1], the general layout of which foresees a mixed compression scheme: RF compression in the photo-injector and one single stage magnetic compression at $1 \mathrm{GeV}$ up to the final peak current of $2.5 \mathrm{kA}$.

Applying velocity bunching in the SPARC photoinjector with low charge bunches (about $15 \mathrm{pC}$ ) will allow the production of ultra-short electron bunches, in the range of a few $\mu \mathrm{m}$ rms bunch length, fully synchronized to the Ti:Sa laser pulses. A further upgrade of the laser system to produce multi-TW pulses, by means of a third stage of amplification and an under vacuum pulse compressor, will allow to conduct laser wake-field plasma acceleration experiments with external injection of high quality ultra-short bunches into the plasma wave, for ultra-high gradient acceleration of electron beams.

This naturally projects SPARC as a more general test facility to conduct advanced beam physics and new acceleration technique experiments at LNF in the near future.

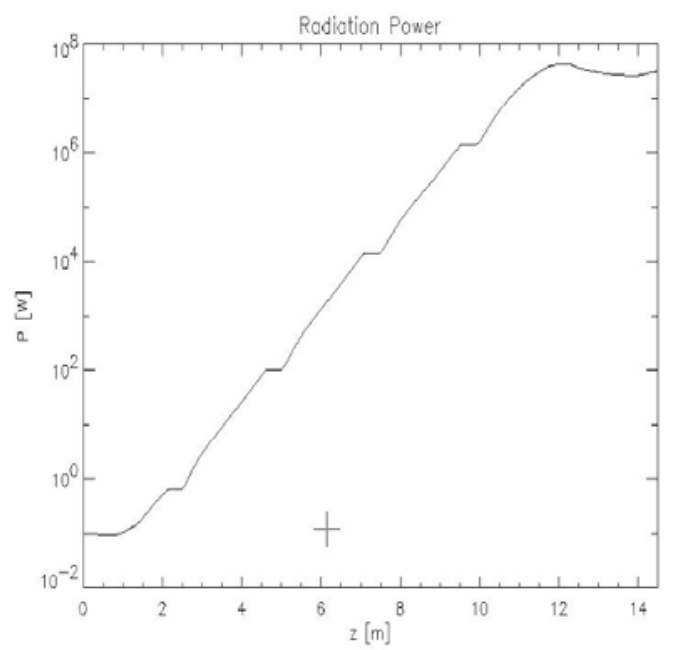

Figure 4: FEL radiation power growth along the undulator

\section{REFERENCES}

[1] D. Alesini et al., Conceptual design of a High Brightness Linac for Soft X-Ray SASE-FEL Source, Proceedings of the FEL-2002 Int. Conference

[2] M. Ferrario et al., Recent Advances and Novel Ideas for High Brightness Electron Beam Production based on Photo-Injectors, INFN Rep. LNF-03/06 (P), May 2003

[3] L. Serafini and M. Ferrario, Velocity Bunching in Photo-Injectors, AIP CP 581 (2001) 87

[4] D.T. Palmer, PhD. Thesis, Stanford University

[5] M. Biagini et al., Beam Dynamics Studies for the SPARC Project, this conference 